\title{
La violencia laboral en la administración pública argentina
}

\author{
Scialpi, Diana*
}

\section{Resumen}

Actualmente, los organismos internacionales de salud pública reconocen a la violencia como un obstáculo para el desarrollo de las naciones y una amenaza para la salud pública. En virtud de ello, la conceptualización, medición y análisis de las conductas violentas en diferentes contextos y la comprensión de sus causas y factores asociados, fueron establecidas como tareas urgentes encaminadas a orientar las acciones de salud pública en lo referente a la prevención de la violencia y la atención a las víctimas y agresores. Por otra parte, el abordaje del fenómeno de la violencia en el trabajo, convoca la atención de los expertos de Europa y Estados Unidos, desde hace sólo una década. Una investigación exploratoria "Violencias en la Administración Pública. Casos y Miradas para pensar la Administración Pública Nacional como ámbito laboral" (Scialpi, 1999) verifica que la conducta violenta es perpetrada, en democracia y en las instituciones estatales, por quienes tienen la responsabilidad de proteger a todos los ciudadanos. La transformación de la fuente de protección en fuente de terror, produce efectos mucho más devastadores por cuanto se convierte en modelo autorizado, además de resultar condición necesaria para el desarrollo permanente de la corrupción. Existe una íntima relación entre violencia político-burocrática, sufrimiento personal (impacto en la salud física y psíquica de los agentes públicos) resultados de la gestión pública, corrupción y resquebrajamiento del sistema democrático y el Estado de Derecho. El presente artículo, producto de una experiencia de observación-reflexión participante, conjuntamente con el análisis de una muestra intencional de casos, aspira propiciar la reflexión colectiva para revertir situaciones concretas que afectan potencialmente a todos los empleados públicos del país, que representan el 14,4\% de la población económicamente activa (PEA) ocupada de Argentina. Como resultado, se presenta la discusión conceptual de términos de reciente generación y se identifican conductas violentas típicas que permitan orientar acciones preventivas de salud pública.

Palabras clave: Violencia laboral, administración de personal, salud pública, violencia institucional, gerencia pública.

Recibido: 01-11-16 . Aceptado: 02-02-20

Socióloga y Especialista en Planificación y Gestión de Políticas Sociales de la Universidad de Buenos Aires. Es docente de postgrado del Instituto Universitario de la Fundación ISALUD e Integrante de la Planta Permanente de la Jefatura de Gabinete de Ministros de la Nación.

E-mail: dscialpi@fibertel.com.ar 


\section{Worker Violence in Argentine Public Administration}

\section{Abstract}

International public health organisms are now recognizing violence as an obstacle to national development and a threat to public health. Because of this, conceptualization, measurement and analysis of violent conducts in different contexts and the understanding of its causes and associated factors were established as urgent actions directed towards orienting public health problems in relation to the prevention of violence and attention for victims and victimizers. On the other hand, the approach to the problem of violence at work attracted the attention of experts in Europe and the United States as of only a decade ago. The exploratory research VIOLENCE IN PUBLIC ADMINISTRATION.: CASES AND VIEWPOINTS ORIENTED TOWARDS NATIONAL PUBLIC ADMINISTRATION IN THE LABOR FIELD (Scialpi, 1999) verified that violent conduct is perpetuated in democracy and in state institutions by those who are responsible for the protection of citizens. The transformation of protection sources into sources of terror produces many more devastating effects since it is converted into an authorized model, and also results in a condition necessary for the permanent development of corruption. There is an intimate relation between bureaucratic-political violence, personal suffering (the impact of public agents on physical and psychiatric health), results of public management, corruption and democratic system breakdowns, and a state of legal rights. This article, which is the product of experience of participative observation and reflection, together with an analysis of an intentional sampling of cases, hopes to offer collective reflection in order to address concrete situations that potentially affect all public employees, which represents $14.4 \%$ of the economically active population in Argentina. As a result, a conceptual discussion is presented in terms of recent developments and typical violent conducts are identified allowing us to orient preventive public health measures.

Key words: Labor violence, personnel management, public health, institutional violence, public management.

\section{Introducción}

Corre el mes de febrero de 2002. En el orden planetario -a partir del 11 de Septiembre de 2001- se plantea la lucha entre estado de derecho y estado de policía y la Argentina desnuda ante el mundo la profundidad de su crisis institucional. En este contexto intentar reflexionar sobre la violencia laboral en nuestros organismos públicos, puede resultar -para atgunos- un cometido de cuestionable importancia.

No obstante, y apelando al necesario equilibrio para reconocer otras prioridades, considero oportuno y relevante in- sistir en un tema escasamente frecuentado por los analistas sociales y los especialistas en estudios laborales y de gerencia pública: la violencia en la gestión de personal de los organismos y las instituciones estatales, en democracia. Es menester, a mi juicio, instalar la temática en la agenda académica y pública y contribuir a su construcción como problema público y objeto de políticas. En esta introducción me permitiré primero, hacer un poco de historia y buscaré explicitar, a partir de un recorrido personal, el sentido de mi trabajo actual.

La mía es sin duda una mirada subjetiva, como la de todos los sujetos, sea- 
mos o no analistas sociales y más allá de que objetivar lo subjetivo sea el cometido de las ciencias sociales. Me atrevo a decir que tal vez en esa subjetividad deba hallarse el valor de mis reflexiones, ya que se trata de la mirada de una socióloga, especializada en Planificación y Gestión de Políticas Sociales, que trabaja -desde 1986- en calidad de personal permanente del sector público nacional argentino y experimenta, padece, observa, analiza y describe ese territorio desde adentro.

Mi experiencia laboral cotidiana me ha colocado, muchas veces, frente a situaciones de trabajo de evidente inequidad. Percibí la violencia y la sentí en carne propia. Por otra parte, desde $1997 \mathrm{mi}$ condición de docente de la Maestría de Salud y Seguridad Social del Instituto Universitario de la Fundación ISALUD, me ha acercado al enfoque de diversos problemas sociales - la violencia entre otros- desde la perspectiva de los organismos internacionales de salud pública. Esta circunstancia fortaleció mi arraigada solidaridad con las amarguras de mis compañeros de tareas y aún con las de otros trabajadores de la Administración Pública Central Argentina, cuyas dolorosas vivencias compartí en los típicos comentarios de pasillo.

Sin duda, estos antecedentes han contribuido a que observara con mayor detenimiento los comportamientos y las actitudes de los agentes públicos, obligados a trabajar y convivir con la irracionalidad colectiva de "Un país al margen de la ley" según lo denominó un memorable libro de Carlos Nino, en 1992. En la última década he adquirido la convicción de que, precisamente, ese tradicional desprecio de los argentinos por la ley, histo- riado por el jurista Nino, es el que pone en riesgo la salud física y mental de los servidores públicos.

De esto nadie hablaba. Nadie se atrevía a develar esta situación. Todos deplorábamos estas prácticas pero...dejábamos hacer. Nos faltaba -a administrativos, técnicos, profesionales y consultores expertos- la necesaria autoridad social para ejercer -en plena democracianuestros derechos laborales y reclamar ante las autoridades tanto el cumplimiento estricto de la normativa vigente como la modificación de las normativas irracionales o inequitativas de la Administración Pública.

La ingobernabilidad -como sostiene Carlos Almada López (2001)-, es un concepto límite que se presenta cuando el estado de derecho se diluye y se rompen los vínculos entre quienes ejercen el poder público y los integrantes de una comunidad política dada. En cambio la gobernabilidad ha de entenderse como una responsabilidad colectiva, que no atañe solamente a los órganos estatales, pues la seguridad jurídica, la estabilidad política y la cohesión social han de ser entendidos como valores a tutelar por toda la población.

Con esa convicción me propuse visibilizar la violencia en la gestión de personal de la Administración Pública Central Argentina, violencia normalizada, naturalizada, invisibilizada y consentida, por todos sus integrantes. "La violencia, es cierto, puede bastar para instituir nuevas relaciones sociales, pero parece no ser suficiente cuando se trata de reproducirlas de un modo durable. El consentimiento es la parte del poder que los dominados agregan a la que los dominantes ejer- 
cen directamente sobre ellos" (Godelier, 1984:23) Investigué sin apoyo económico o institucional alguno, sobre la base de una muestra intencional de casos (con expedientes), propia de los estudios exploratorios. Conté con la indispensable colaboración de un experto abogado administrativista -el Dr. Luis Cantelmo- y con la invalorable perspectiva de muchos otros especialistas, bibliotecólogos, politólogos, psicólogos, antropólogos. A partir de esa visibilización, me siento responsable y comprometida en la promoción de un proceso de discusión colectiva sobre la violencia de nuestra Administración Pública, violencia hacia adentro -hacia los propios agentes, trabajadores, funcionarios- que se traduce a su vez en violencia hacia fuera, hacia el público entendido como beneficiario o usuario de la gestión pública. La coyuntural y oportuna perspectiva de los organismos internacionales de salud pública es más que propicia. Veamos por qué.

Actualmente, los organismos internacionales de salud reconocen a la violencia, en sus diferentes manifestaciones, como un obstáculo para el desarrollo de las naciones y una amenaza para la salud pública. En informes especializados, dichos organismos destacan que la violencia ha dejado de enfocarse indirectamente y de esconderse tras apelativos como "cuestiones de salud pública controvertida" y "hecho inevitable de la vida" para reconocerse como una "epidemia soslayada", que pronto superará a las enfermedades infecciosas como causa principal de morbilidad y mortalidad prematura en todo el mundo. Más aún, han estimado que los actos de violencia con- tribuyen al $15 \%$ de la carga mundial de enfermedad, pero advierten que no existen indicadores adecuados para medir su impacto total. Las tasas de mortalidad por homicidio y suicidio, que son fáciles de obtener y sirven para hacer ciertas comparaciones entre países, no incluyen las muertes no registradas relacionadas con la violencia. Por consiguiente, la conceptualización, medición y análisis de las conductas violentas en diferentes contextos aparecen, hoy, como tareas urgentes. Los informes de la Organización Panamericana de Salud (Fournier et al, 1999) sostienen que es preciso profundizar en el conocimiento y la comprensión de la compleja trama de factores políticos, socioeconómicos, psicosociales y culturales que se asocian con la violencia y fundamentar un enfoque integrado de prevención y atención a las víctimas y agresores. Para ello, la promoción y realización de investigaciones y el mejoramiento de los sistemas de información y registro, resultan prioritarios.

En América Latina, este impulso de los organismos internacionales, de estudiar las conductas violentas en diferentes contextos, se orienta primero al análisis de la violencia física interpersonal (en la familia, en la pareja, hacia los niños, hacia personas ajenas a la familia) Un claro ejemplo de ello es el Estudio Multicéntrico sobre Actitudes y Normas Culturales frente a la Violencia -proyecto ACTIVAque es una iniciativa interamericana de cooperación entre la Organización Panamericana de la Salud y centros de investigación de siete países. El estudio se efectuó en ocho áreas metropolitanas de América Latina y España. Quedó exclui- 
da la evaluación de la violencia ejercida por instituciones y organizaciones de diferente índole, la violencia psicológica y la violencia sexual. Tampoco se consideraron las manifestaciones de violencia en el lugar de trabajo ni en las escuelas. Por último, la violencia física interpersonal, fue entendida, a los fines del estudio, sólo como el uso intencional o la amenaza de usar la fuerza física que ejerce una persona para causar daño físico a otra, a un grupo de personas o al patrimonio personal o social (Filho, 1999).

Al mismo tiempo, el problema de la violencia en el lugar de trabajo comienza a convocar la atención de los expertos, en Europa y en EEUU desde hace menos de una década. Los conceptos de violencia laboral, mobbing, acoso moral y psicoterror, como fenómenos de patología laboral, se identifican recién a partir de 1996.

A la luz de la perspectiva de los organismos internacionales de salud, mi razonamiento es el siguiente: $\mathrm{Si}$ el estudio de las conductas violentas en diferentes contextos es considerado tarea urgente, más urgente aún resulta estudiar, visibilizar, conceptualizar y medir la violencia, cuando es perpetrada en democracia y en las instituciones estatales, por quienes, de acuerdo a Sluzky (1994), tienen la responsabilidad social y legal de cuidar a los ciudadanos, de mantener el orden en su mundo, de preservar la estabilidad y predictibilidad de sus vidas: el Estado a través de sus agentes. La violencia así instalada en la Administración Pública, es equivalente a la violencia del padre en un contexto de violencia familiar. Vicia y desvirtúa la autoridad necesaria para conducir los organismos públicos, circunstancia de la que nos avergonzamos muchos argentinos.

A mi juicio, existe una íntima relación entre sufrimiento personal (impacto en la salud física y psíquica de los agentes públicos) resultados de la gestión pública y resquebrajamiento del sistema democrático y del Estado de Derecho. Por encima de esta asociación, para mí evidente, emerge su funcionalidad indiscutible: La "violencia político-burocrática", como la denominé, aparece como condición necesaria para el desarrollo de la corrupción, fenómeno éste que comienzan a combatir los propios organismos multilaterales de crédito y las grandes corporaciones multinacionales que también se sienten amenazados por estos procedimientos. Por añadidura, perpetradas en el territorio de la Administración Pública, estas conductas violentas construyen un modelo autorizado (Coria, 1994) y en ello debemos hallar, a mi juicio, su máxima peligrosidad.

Las notas y reflexiones que siguen a continuación forman parte de una investigación que se propone reunir las principales consideraciones actuales referidas a la violencia en el lugar de trabajo, con especial atención a la violencia en el empleo público. Esto equivale a poner la mirada -en una primera etapa- sobre la violencia hacia adentro, hacia los propios integrantes, agentes, trabajadores y funcionarios de la Administración Pública.

Una segunda etapa de la investigación, se propone poner el foco en las violencias hacia afuera, que son las que experimentan los ciudadanos en general, entendidos como usuarios o beneficiarios de los servicios que prestan las instituciones estatales. 
El texto que presento aquí, incluye breves referencias a la violencia en el empleo público de los Estados Unidos y al fenómeno del acoso moralen el servicio público de Francia, analizado por $\mathrm{Hi}$ rigoyen (2001). Asimismo, ofrece datos y reflexiones contenidas en un trabajo previo (Scialpi, 1999) y da mínima cuenta de la existencia de algunos proyectos legislativos argentinos y extranjeros y de jurisprudencia extranjera en la materia. Por último informa sobre algunas propuestas de acción para atender y revertir el fenómeno.

Agradezco el estímulo permanente de la Dra. Diana Maffia, Defensora Adjunta del Pueblo de la Ciudad Autónoma de Buenos Aires, a mi iniciativa de implementar un programa de detección, prevención y atención de situaciones de violencia en la Administración Pública y el invalorable apoyo del Lic. Jorge Corsi -Director de la Carrera de Especialización de Posgrado en Violencia Familiar de la Facultad de Psicología de la Universidad de Buenos Aires- para inaugurar un Curso de Posgrado y una materia optativa sobre "Violencia Laboral e Institucional", a partir de septiembre del corriente año 2002, conjuntamente con la Dra. Hilda Rubio. Aprecio lo aprendido de mis colegas: Matilde Mercado, especializada en cuestiones de Género en el Trabajo e Inés Izaguirre (1998), cuyos trabajos sobre conflicto social y violencia me resultaron tan esclarecedores. Finalmente agradezco al Dr. Bernardo Kliksberg su apoyo a mi trabajo y a la Revista Venezolana de Gerencia la invitación a participar de esta publicación.

\section{La violencia laboral de la administración pública argentina como problema social}

El sector público ha experimentado durante la década de los años noventa, importantes transformaciones que remiten a procesos más amplios de cambio en las funciones y en las estructuras de los Estados. Según Oszlak (2000:1) "la Argentina es, posiblemente, el país en el que se han producido durante los años 90', las transformaciones más radicales en la configuración, tamaño y papel del estado nacional. El caso argentino ha despertó un lógico interés de parte de los organismos multilaterales de crédito por difundir esta experiencia y señalarla como un modelo a imitar por otros países embarcados en procesos de reforma y modernización estatal, aún cuando no existe hasta la fecha una comprobación fáctica que permita efectuar afirmaciones comparativas". Más aún, Oszlak (2000) se preguntaba si el modelo que emerge marcaría el destino de otros estados nacionales o sería un simple modelo suigeneris, transición hacia alguna otra forma de estado nacional apropiado para una época de globalización y, simultáneamente de subnacionalización, como la que estamos atravesando.

A mi juicio, sería una tragedia - para cualquier país - imitar una Modernización del Estado y Reformas Administrativas como las realizadas en la República Argentina, para orientar el destino de sus sociedades. Muchos de sus procedimientos fueron (y son aún) a tal extremo des- 
tructivos que evocan las reflexiones del politólogo Guillermo O'Donnell (1997), quien, en una entrevista al diario Clarín, sentenció que en el caso argentino, había que pensar en la conjunción entre la oligarquía vengativa y la casta de ladrones que entonces avanzó sobre el poder, saqueando empresas públicas y haciendo más que negociados. Según O'Donnell, el Estado no se asumió como Estado ni siquiera para matar y algunas formas criminales que se observan hoy tienen una matriz allí. De modo que, a su juicio, no sólo se destruyó al Estado como instrumento social sino que también se lo gangsterizó.

En otra entrevista en el diario La Nación, el mismo O'Donnell (2000) afirmó que los politólogos argentinos han puesto énfasis en los análisis "macro" y descuidado los escenarios "micro"; y agregó que consideraba necesario hacer una antropología de la democracia, una investigación más atenta a las conductas cotidianas en los micro espacios. A ese expreso objetivo apunta este trabajo. Mi propósito es dejar constancia de algunos mecanismos, procedimientos y conductas violentas de nuestra Administración Pública que son efectivamente nuestra "epidemia soslayada". A mi juicio, reviste carácter no ya de urgencia sino de emergencia prestarles atención, en tanto aparecen en la institución oficial por excelencia. La declarada responsabilidad de promover y contribuir a la configuración de "Un gobierno más efectivo y menos costoso" (Gore, 1995) requiere, a mi entender, como punto de partida, encarar la tarea ineludible de reflexionar en torno a la cultura de trabajo en el empleo público argentino. Imagino que la potencialidad de este análisis está vinculada a la posibilidad de difundir diversas reflexiones y argumentos y propiciar la de-construcción y re-construcción de los mecanismos cotidianos que modelan los modos de pensar, de sentir, y aún de enfermarse de los trabajadores de la Administración Pública Argentina, generando modalidades de relación institucionales e interpersonales profundamente antidemocráticas.

Aclaro que este texto no incluye análisis alguno de causas históricas o coyunturales, políticas, económicas, sociales y culturales, que incorporé sintéticamente en el trabajo publicado en 1999. Su relevancia y necesario esclarecimiento, exceden los objetivos de este artículo. El problema aquí y ahora, es visibilizar e identificar conductas violentas para orientar las acciones de salud pública referidas a la prevención de la violencia en la Administración Pública en particular y en las instituciones estatales en generaly a la atención a las víctimas y agresores. Se trata de contribuir a asegurar la construcción de un estado más saludable y en consecuencia, más racional, más democrático, más transparente, más justo, más humano.

No obstante, debemos recordar siguiendo a Horton (1987), que la sola existencia de ciertas condiciones sociales objetivas, no es condición necesaria y suficiente para su reconocimiento como problema social. Es preciso, además, que esa condición afecte a un número importante de personas de un modo considerado inconveniente y que la gente crea que puede y debe hacer algo para corregirla mediante la acción social colectiva, en virtud de que es considerada socialmente indeseable. En la Argentina esta 
condición afecta a un número importante de individuos. El estado nacional empleaba en 1998, a 291235 agentes que representan al 1,8\% de la Población Económicamente Activa (PEA) del país y en 2000, el Poder Ejecutivo Nacional ocupaba 255432 agentes, incluyendo al personal de las fuerzas armadas $y$ al de seguridad (Oszlak, 2000). Pero la población total potencialmente afectada son los empleados públicos de todo el país -1.813.381conjunto de asalariados que representan el $14,4 \%$ de la Población Económicamente Activa, PEA, ocupada del país (SIEMPRO-INDEC, 2000). Para agregar fundamentos al reconocimiento de esta problemática como problema social, coincido con Araujo (2000) quien afirma que los problemas públicos no existen por sí mismos como meros fenómenos objetivos, sino que son construidos por actores que se mueven en distintos escenarios, intercambiando y confrontando discursos que se sustentan en marcos interpretativos variados.

Es posible encontrar antecedentes alentadores. En el tema de la violencia, como en otros fenómenos sociales, hay un largo camino que va desde el momento en que se identifica ( $y$ se visibiliza) una modalidad de violencia como problema social hasta que se elaboran marcos teó ricos comprensivos y se toman decisiones para su atención y tratamiento. Así por ejemplo, hace veinte años, poco o nada hablábamos de violencia familiar en la Argentina. No obstante, el fenómeno existía. Desde 1984, un proceso de construcción social llevó primero a hacer visible el problema, después a instalarlo socialmente y finalmente a impulsar prácticas reparadoras que incluyen estrategias colectivas e intervenciones de diversas disciplinas -jurídicas, médicas, psicológicas, sociales, culturales- destinadas a prevenir, atender y reparar situaciones de violencia familiar. Actualmente, la Universidad de Buenos Aires, cuenta con una Carrera de Posgrado de Especialización (interdisciplinaria) en Violencia Familiar. En materia legislativa también se han dictado leyes de amparo.

Cabe preguntarse ¿Por qué no podemos generar, del mismo modo, estrategias para la violencia en diferentes ámbitos laborales, comenzando por la violencia en las instituciones estatales que es la que instala un modelo autorizado? Es obvio que por encima de las particularidades observables en cada sector de actividad, la conducta violenta en ámbitos laborales es verificable, aunque naturalizada y ocultada como ocurrió con la violencia familiar. Más naturalizada y menos denunciada, aún, es la Violencia en la Administración Pública entendida como ámbito laboral donde es perpetrada en democracia, por quienes tienen el deber social, legal y administrativo de proteger a todos los ciudadanos. Su visibilización y su denuncia, adquieren carácter de emergencia social. Su tratamiento y corrección también.

Creo firmemente que eludir esta parte sustancial de la conflictividad humana, subestimarla o minimizarla es una forma de justificación. Más aún, no exponerla equivaldría a incurrir -a mi entender- en una "doble victimización" (Corsi, 1994) y contribuir a la perversión del sistema, proceso, como afirma Hirigoyen (1999), que está en la base del funcionamiento de la mafia o de los regímenes totalitarios. 


\section{Modificaciones del perfil de la violencia en el trabajo}

Filho (1999) señala que el estudio de la violencia interesa por su efecto deletéreo en las condiciones generales de salud y bienestar de las poblaciones. Los problemas de la salud pública deben abordarse desde la perspectiva de la sociedad. Ello exige diseñar políticas que tomen en cuenta los factores que determinan la salud en este nivel y que reconozcan el carácter esencialmente político y social de los problemas de salud de las colectividades.

También corresponde distinguir la diferencia entre tratar la violencia como un problema de salud pública y aplicar el enfoque de la salud pública al problema de la violencia. Los que proponen la última opción suelen identificar al enfoque de la salud pública con el enfoque de riesgo, según el cual los factores determinantes del carácter sociocultural suelen analizarse a partir de unas pocas variables tratadas como si fueran atributos de individuos y no de grupos.

Cuando hablamos de fenómenos de violencia en ámbitos laborales estamos aludiendo a una problemática que comprende fenómenos y conductas violentas recurrentes, no-episódicos ni únicos. Sin embargo, no ha sido este tipo de fenómenos repetidos y prácticamente naturalizados, los que han trascendido como ejemplos de violencia en el trabajo, sino determinadas tragedias únicas y singulares. De acuerdo al primer informe de la Organización Internacional del Trabajo (único en su género hasta el presente y sin traducción al español): "Violence at work" (Chappell y Di Martino, 1998) fue- ron dos tragedias ocurridas hace sólo cinco años, las que han estimulado la atención internacional prestada a la violencia en el lugar de trabajo y las que han impulsado su abordaje como tema de estudio, investigación y debate.

Los dos sucesos fueron, el primero: la tragedia de la Escuela Primaria de Dunblance, Escocia, en marzo 1996. El asesinato de docentes y veinte niños, llevó al gobierno del Reino Unido a reformular las leyes de tenencia de armas de fuego y a rediseñar estrategias de seguridad en las escuelas. El segundo suceso fue la masacre en el Parque Nacional de la Isla de Tasmania, Australia, en abril 1996, donde fueron asesinadas treinta y cinco personas, entre ellas muchos empleados del Parque. Ambos son ejemplos raros y extremos de intrusión de violencia en el lugar de trabajo que indujeron la redefinición de estrategias de prevención y de investigación. Chappell y Di Martino (1998) manifiestan que el aprendizaje ha sido en extremo doloroso por cuanto no podemos soslayar que ambas fatalidades han dejado una lección a incluir en los antecedentes de toda reflexión y formulación de políticas de prevención de la violencia en el trabajo.

Este primer informe de OIT señala que en los EEUU, el homicidio se ha convertido en la segunda causa del total de muertes ocupacionales, y la primera causa con relación a la mujer. Las cifras del Reino Unido, de Alemania y Japón no son menos llamativas y muestran que el tema trasciende las fronteras de un país determinado. Ningún país puede considerarse ajeno al fenómeno, aunque ciertamente, algunas ocupaciones parecen más expuestas y algunos países más afectados. 
Otro señalamiento del mismo informe es que la variedad de conductas que pueden ser comprendidas dentro del concepto de violencia laboral es tan extensa, la línea fronteriza que separa las conductas aceptables de las que no lo son, es tan vaga y la percepción en diferentes contextos y culturas de lo que constituye violencia, es tan diversa, que resulta un verdadero desafío tanto describir como definir el fenómeno que enfrentamos. Para los expertos autores del informe, la violencia en el lugar de trabajo incluye una amplia gama de conductas que comprende 29 ítems que van desde el homicidio hasta los silencios deliberados.

De lo expuesto hasta aquí, advertimos que, hasta la fecha, existe una zona ambigua, de confusión cuando hablamos de violencia en el lugar de trabajo (aludiendo al ámbito y ubicación espacial del suceso) o cuando hacemos referencia al término violencia laboral. ¿Debemos entender que la injuria, el daño intencional o el peligro, es perpetrado por terceros ajenos al empleo en cuestión? (público, beneficiarios o usuarios en general, o sea individuos) ¿Y/o distinguir la violencia propia de la organización laboral en la que interactúan, primordialmente, empleados y empleador (problema organizacional o estructural)?

Las tragedias mencionadas, de Escocia y Tasmania, se verificaron en un lugar de trabajo, pero corresponde definir y hasta la fecha esta compleja distinción no se ha realizado - si, y cuándo, los riesgos son inherentes al empleo o la ocupación. Huelga decir que la tragedia de las torres gemelas de Nueva York en Septiembre de 2001, supera estas prelimina- res conceptualizaciones de la OIT, de 1998 (Chappell y Di Martino, 1998) que consideramos aquí.

Por último, en materia de violencia laboral comienza a visibilizarse -también desde hace pocos años- otro fenómeno que agrega mayor complejidad al desafío conceptual planteado. Chappell y Di Martino (1998) informan que en los últimos años, denuncias provenientes de países como Australia, Austria, Dinamarca, Alemania, Suecia, Reino Unido y Estados Unidos, dan cuenta de una nueva forma colectiva y sistemática de violencia. Esta consiste en tomar a una persona de "blanco" de "hostigamiento y acoso psicológico persistente", que conllevan críticas, aislamiento, chismes, injurias y ridiculizaciones. El impacto de este tipo de conductas es devastador y en varios países europeos se emprenden acciones colectivas para atender el fenómeno (Sánchez Carazo, 2001).

\subsection{La violencia en el empleo público de Estados Unidos}

La Revista de Administración Pública de los EEUU, publica un informe de Nigro y Waugh, Jr. (1996) que define a la violencia ocupacional o violencia en el lugar de trabajo como un daño intencional, violación u homicidio durante el curso del empleo y afirma que las estadísticas disponibles revelan que este tipo de violencia resulta un riesgo significativo para muchos trabajadores, cuyas consecuencias podrían ser más graves de lo sugerido por los datos. Más aún, señalan que las causas psicológico sociales están siendo exploradas pero que, hasta el mo- 
mento, no se ha realizado ningún esfuerzo sistemático para identificar factores de riesgo que pudieran ser particularmente relevantes para los empleados públicos.

Los mismos autores sostienen que hay razones para creer que el sector público (norteamericano) está siendo cada día más amenazado por la violencia antigubernamental - clientes frustrados, grupos terroristas con motivos políticos, individuos que confrontan con la burocracia. El empleo público representa un $18 \%$ de la fuerza laboral de EEUU, los trabajadores públicos son las víctimas del $30 \%$ de los casos de violencia en el trabajo entre los años 1987-1992. Por último, la revisión del estado del conocimiento en materia de violencia ocupacional en los Estados Unidos, permite deducir que la guía que ofrece a los empleadores públicos es limitada. Como conclusión, se admite que los requerimientos de investigación habituales, requieren la conformación de una base de datos nacional sobre Occupational Violent Crime (OVC) que incluya información sobre variables psicológicas, sociales, organizacionales y otras.

Resulta de particular interés a los efectos de este trabajo, que Nigro y Waugh Jr. (1996) indiquen que los empleadores públicos deberían asumir el liderazgo en el esfuerzo de prevenir la OVC y enfrentar sus consecuencias. Sus conclusiones destacan dos cuestiones importantes. La primera: La mayor parte de los empleadores - públicos o privados se resisten a la idea de tratar la Violencia Ocupacional como un problema organizacional (estructural) (O'Donnell, 1999). La segunda: Los estudios empíricos sobre violencia ocupacional en los EEUU son limitados. Una tercera cuestión que me permito agregar, y resultante del mismo informe, es la identificación de profesiones peligrosas. Paradójicamente los trabajadores de la salud, parecen llevar la delantera en materia de ataques y violencias en el lugar de trabajo.

\subsection{Vacío estadístico y falta de acuerdo conceptual}

Hasta la fecha, no existen estadísticas confiables sobre violencia en el trabajo. Este vacío es explicable, en parte, por la falta de acuerdo conceptual, y también, por la ausencia de responsables específicos en la mayoría de los países. No hay registros. Los datos compilados refieren por un lado, a Higiene y Seguridad en el Trabajo y por otro a informaciones sobre crímenes provenientes de la justicia penal asociados a violencia.

En la actualidad, una de las más importantes fuentes de información oficial sobre violencia en el trabajo es la que proviene de la Encuesta Internacional del Crimen "IC (V) S", un estudio multinacional comparativo que comprende, hasta ahora, a más de cincuenta países. La encuesta es coordinada por un equipo de trabajo internacional, compuesto por representantes del Ministerio de Justicia de Holanda, el Instituto Nacional Interregional de Investigaciones Criminales y Judiciales (UNICRI) y la Home Office del Reino Unido. Más allá de estas dificultades, el informe de Chappell y Di Martino (1998), ofrece alguna información estadística. En un cuadro de resultados a tres columnas, aparecen datos de más de treinta países y regiones distribuidos según diferentes tipos de violencia: (1) asaltos a varones en los lugares de trabajo, 
(2) asaltos a mujeres en los lugares de trabajo y (3) incidentes sexuales. Argentina ostenta el tercer lugar, con relación a asaltos padecidos por varones en el lugar de trabajo; el primer lugar de la columna de asaltos a mujeres y el segundo lugar en la columna relativa a incidentes sexuales. En ésta última, Francia ocupa el primer lugar.

El desafío actual y la mayor dificultad para realizar cualquier análisis y construir una base de datos estadísticos sobre este tema, provienen de la falta de acuerdo en las definiciones de violencia, de trabajo y de lugar de trabajo. En este sentido, Chappell y Di Martino (1998) afirman que el término violencia y el término agresión se usan indistintamente, siendo el primero más común en el área de la justicia criminal y el segundo más habitual en el campo de la salud. También señalan que, en un intento realizado por comprender y prevenir la violencia, la Academia Nacional de Ciencias de los Estados Unidos definió la violencia o la conducta agresiva, como la conducta llevada a cabo por individuos con la intención de amenazar, atentar o infligir daño físico en otras personas o a sí mismo. Y concluyen que dicha definición excluye la violencia dirigida contra la propiedad (que representa un ítem significativo en el lugar de trabajo) y excluye la violencia de naturaleza psicológica, que comprende el abuso emocional, y la inflicción de ansiedad y miedo. La misma definición excluye el delito del trabajoen sentido amplio, concepto que comprende delitos no-violentos como robo, fraude, desfalco o peculado.

Por último, y como conclusión de este informe de OIT al que vengo hacien- do referencia, Chappell y Di Martino (1998) destacan la importancia de atender este problema e invitan a 1) repudiar la violencia en el trabajo 2) hacerla visible; 3 ) instalarla como cuestión pública; 4) discutir estrategias concretas de acción para atenderla y 5) convocar a la acción internacional.

\subsection{Algunos conceptos de reciente generación}

\section{a. Mobbing}

El término mobbing, (proveniente de la lengua inglesa, del verbo "to mob" (atacar, asaltar) y de la etología, en tanto refiere al comportamiento de algunos animales que, generalmente, acosan minuciosamente a un miembro del grupo para alejarlo) agrupa una diversidad de comportamientos que tiene entre ellos un fondo común: la modalidad agresiva y vejatoria tendiente a la eliminación de un trabajador de la empresa. Una suerte de terror psicológico ejercido en el lugar de trabajo. Las formas en las que suele presentarse son variadas y de las más sutiles: el aislamiento físico del trabajador, la marginación a través de la hostilidad y la falta de comunicación; la difusión de conceptos peyorativos; la asignación de tareas humillantes, descalificadoras o de muy difícil concreción; el menoscabo de la imagen del empleado frente a sus colegas, clientes o superiores, los cambios continuos, entre otros.

Todas estas modalidades de acoso a la identidad e integridad psíquica del trabajador, obviamente no constituyen, por sí mismas, ninguna novedad. Probablemente, lo que ha concitado la atención 
de los juristas europeos -y en definitiva la conceptualización misma del "mobbing"ha sido el análisis del problema sobre la base de la repetición de estos hechos muchas veces difusos -en un lapso suficientemente prolongado. La importancia del concepto "mobbing", consiste en haber encontrado un nombre y una definición bajo los cuales es posible agrupar en una categoría unificante- una serie de comportamientos basados sobre la violencia moral y la persecución psicológica que tienden a desacreditar, discriminar o dañar al trabajador (Revista Derecho del Trabajo, 2000).

Por otra parte, otra característica del mobbing es que la estrategia de persecución puede provenir de sujetos diversos. El mobber (o sujeto activo del mobbing) puede ser tanto uno o más colegas del trabajador que pretenden excluirlo por resultarles incómodo, (incluso por su alto nivel de eficiencia) como la misma empresa, en cuyo caso el fenómeno es conocido como bossing o mobbing vertical. En estos casos, la presión psicológica del empleador está acompañada por los side mobbers (colegas, superiores, gerentes de recursos humanos, etc.) que aún no participando directamente en el acoso, por razones de posicionamiento dentro de las preferencias jerárquicas, excluyen o evitan todo tipo de solidaridad con la víctima. El mobbing desemboca generalmente en una renuncia del trabajador a su empleo no sin antes haber sufrido, seguramente, patologías psíquicas y psicosomáticas.

En Italia existe jurisprudencia sobre esta materia y hasta la fecha se han presentado cinco proyectos de ley (Revista Derecho del Trabajo, 2000). Por su parte, y a la hora de revisar este texto, asisto en Madrid, a la Primera Jornada Nacional sobre Acoso Psicológico en el Trabajo, con presentación de sentencias de jurisprudencia del Tribunal Supremo y distribución de Proposiciones de Ley presentadas por el Congreso de los Diputados, sobre el derecho a no sufrir acoso moral en el trabajo y la Propuesta Orgánica para tipificar en el código Penal al acoso moral en el trabajo (Boletín Oficial de las Cortes Generales España, 2001).

Por último Heinz Leymann psicólogo del trabajo e investigador de procesos como los considerados, citado por Chappell y Di Martino, (1998) y por Hirigoyen (1999 y 2001) utiliza, para los mismos, la denominación "psicoterror". Un estudio de Leymann en 1990 citado por Hirigoyen (2001), establece que en Suecia, el hostigamiento psicológico en el trabajo origina entre el $10 \%$ y el $15 \%$ de los suicidios registrados.

\section{b. El "acoso moral" y su visibili-} zación en el servicio público francés

En años recientes, en Francia se vienen advirtiendo los efectos negativos de la violencia laboral sobre la salud de las personas. No obstante, si bien se admite un costo económico, éste no se ha evaluado con exactitud. Hirigoyen (1999) acuñó el concepto acoso moralpara describir un proceso de maltrato psicológico en el que un individuo puede conseguir hacer pedazos a otro y el ensañamiento puede conducir incluso a un verdadero asesinato psíquico. Según la experta, por acoso en el lugar de trabajo hay que entender cualquier manifestación de una conducta abusiva y, especialmente, los 
comportamientos, palabras, actos, gestos y escritos que puedan atentar contra la personalidad, la dignidad o la integridad física o psíquica de un individuo, o que puedan poner en peligro su empleo, o degradar el clima de trabajo.

Hirigoyen (1999) quien participó con el FBI de los EEUU, en estudios sobre asesinos en serie, sostiene que cuando el proceso de acoso en la empresa se instaura, la víctima es estigmatizada: se dice que el trato con ella es difícil, que tiene mal carácter o que está loca. Se considera que su personalidad es la responsable de las consecuencias del conflicto y la gente se olvida de cómo era antes o de cómo es en otro contexto. Más aún, las víctimas, al principio y contrariamente a lo que los agresores pretenden hacer creer, no son personas afectadas de alguna patología o particularmente débiles. Al contrario, el acoso empieza cuando una víctima reacciona contra el autoritarismo de un superior y no se deja avasallar. Su capacidad de resistir a la autoridad a pesar de las presiones es lo que la señala como blanco.

Hirigoyen (1999) advierte que la empresa en su conjunto se puede convertir en un sistema perverso cuando el fin justifica los medios y cuando la organización o la institución está dispuesta a todo - incluso a destruir a sus empleados con tal de alcanzar sus objetivos. En este caso, la mentira desencadena el proceso perverso en el mismo nivel de la organización del trabajo. La experta ofrece recomendaciones para las víctimas, que incluyen consejos básicos para el fortalecimiento psicológico y estrategias para hacer que intervenga la justicia. Por último Hirigoyen analiza el acoso moral en los ámbitos laborales y da cuenta pormenori- zada de las características que asume el acoso moral en el servicio público francés. "Los métodos de acoso son más perniciosos ahí (en el sector público) y desembocan en resultados dramáticos para la salud y también para la personalidad de las víctimas" (Hirigoyen, 2001:110). En virtud de que el sector público está relacionado con el bien público, que supone la existencia de una lógica de su misión, los abusos, allí, resultan todavía más chocantes. Según Hirigoyen (2001) en el servicio público, el acoso moral no está relacionado con la productividad sino con los juegos de poder. En este caso, a su juicio, no se puede des-responsabilizar a las personas responsabilizando al sistema capitalista y a la globalización. Sólo se puede asociar el acoso moral a una dimensión psicológica fundamental: la pulsión que lleva a los individuos a controlar al otro y a querer avasallarlo.

Los sectores incluidos en el análisis del servicio público francés, son la Administración Pública (nacional, comunal y departamental), las fuerzas armadas, el sector médico-social, el sector educativo y la investigación. Respecto de esta última, Hirigoyen señala que "los procedimientos de acoso moral, entre los investigadores, son incluso mecanismos de supervivencia, pues entre ellos, muy pocos llegarán a hacerse conocer. Como los cargos son bien remunerados, ello lleva a luchas feroces 0 a rivalidades sangrientas. Sólo el mejor (o el que tiene más suerte) logra la notoriedad. El que logre tener éxito podrá mirar a los demás desde arriba, lo cual no lo eximirá de seguir desconfiando de ellos. Los que quedan atrás, acumulan dolor y rencor" (Hirigoyen, 2001: 125). 


\section{La violencia institucional de la administración pública argentina}

La palabra violencia, evoca de inmediato, imágenes que tienen que ver con el empleo de la fuerza material: golpes, hechos de sangre, estallido, combate. Pero la violencia, en términos generales es una forma de ejercicio del poder mediante el empleo de la fuerza (no sólo) física, (sino también) psicológica, emocional, económica, política. En sus múltiples manifestaciones implica -como señala Corsi (1994)- la existencia de un arriba y un abajo, reales o simbólicos, que adoptan habitualmente la forma de roles complementarios: padre-hijo, hombre-mujer, maestro-alumno, patrón-empleado, joven-viejo. El empleo de la fuerza se constituye en un método posible para resolver conflictos como un intento de doblegar la voluntad del otro, de anularlo precisamente en su calidad de "otro". La violencia implica una búsqueda de eliminar los obstáculos (las normativas) que se oponen al propio ejercicio del poder, mediante el control de la relación obtenido a través del uso de la fuerza ( abuso de poder).

Por su parte, Sluzky (1994) sostiene que por violencia política debe entenderse "aquella violencia perpetrada por quienes tienen la responsabilidad social y legal de cuidar a los ciudadanos, de mantener el orden en su mundo, de preservar la estabilidad y predictibilidad de sus vidas: El Estado, a través de sus agentes tales como la policía, las fuerzas armadas. El mismo autor, argumenta que su propia definición de violencia política, que subraya la transformación de la fuente de protección en fuente de terror en un contexto engañoso, lejos de ser específica, borra buena parte de la distinción entre la (macro) violencia política y la (micro) violencia familiar y permite abarcar un amplio espectro de situaciones. La calidad siniestra y el efecto traumático devastador de la violencia política, institucional y familiar, son generados por la transformación del victimario, de protector en agresor, en un contexto que mistifica 0 deniega las claves interpersonales mediante las cuales la víctima reconoce o asigna significados a los comportamientos violentos y reconoce su capacidad de consentir o disentir. Así, la violencia adquiere características devastadoras cuando el acto de violencia es "re-rotulado". La re-rotulación corre por cuenta del violento, quien atribuye a su conducta un significado diferente del que realmente tiene.

Este modelo general de Sluzky (1994) resultó, a mi juicio, un instrumento preciso y oportuno para abordar el territorio de la Administración Pública, entendido como ámbito laboral. En el amplio espectro que va de la (micro) violencia familiar a la (macro) violencia política que menciona el autor, ubiqué, precisamente, situaciones de violencia características de la Administración Pública Argentina. Su modelo me permitió verificar la existencia de diversas modalidades de conductas violentas a las que, genéricamente y previa reformulación de la definición original de Sluzky (1994), denominé violencia político-burocrática, en atención a que es perpetrada en una organización burocrática dirigida por políticos. 


\subsection{La "violencia político-burocrática", una violencia laboral específica}

La violencia político-burocrática es una variante (tipo específico) de violencia política, perpetrada (sobre los agentes del sector público), por funcionarios políticos de la Administración Pública y por funcionarios estables de alto rango (Personal Jerárquico con funciones ejecutivas y personal a cargo) que tienen la responsabilidad social, legal y en este caso, administrativa, de cuidar a los trabajadores del Estado y de cumplir y hacer cumplir las normas administrativas vigentes. Este tipo de violencia político-burocrática es fortalecida y perpetuada, además, por el apoyo de no-decisores que convalidan dichas prácticas (Scialpi, 1999).

En cualquiera de sus muchas variantes, esta violencia política-burocrática tiene un efecto devastador y de largo alcance en quienes han sido y son sus víctimas. Este efecto se deriva de la coexistencia de dos factores, a saber: a) Que la violencia político - burocrática es perpetrada, precisamente por los funcionarios políticos y aún por personal jerárquico de planta y administrativos de carrera de alto rango de la Administración Públi$\mathrm{ca}$, quienes tienen la responsabilidad de respetar y hacer respetar las normas administrativas vigentes. b) Que la transformación del carácter protector en agresor ocurre en un ámbito laboral y en un discurso que destruye o falsea los significados y deniega esta transformación.

La violencia adquiere características devastadoras cuando el acto de violencia es re-rotulado. Por ejemplo, se de- clara: "Esto es organización y capacitación para la eficiencia y la eficacia". El efecto, sea dolor físico, o emocional, es negado o mistificado. Por último, el corolario de valores es redefinido: Se dice: "De esta manera estamos modernizando el Estado" y se practican una serie de actos como los que aparecen enumerados en el recuadro siguiente. La re-rotulación corre por cuenta del violento, quien atribuye a su conducta un significado diferente del que realmente tiene. Así construidas, podemos visibilizar diferentes modalidades de violencias que enuncio, a continuación (en negrita) y que ilustro con ejemplos, sólo en algunos casos.

a) Abierta violación de normas vigentes por parte de quienes las crean $y$ por parte de quienes deben hacerlas cumplir.

b) Coexistencia de numerosos escalafones vigentes o sistemas de empleo diferentes. A este respecto Oszlak (1994) sostiene que la Argentina es el único país latinoamericano donde coexisten al menos cuatro formas diferentes a través de las cuales los gobiernos de la región han intentado introducir cambios para mejorar la calidad de sus administraciones públicas. En el mismo sentido la Dirección de Estudios e Investigaciones del Instituto Nacional de la Administración Pública (Revista Temas, 1995) verificó la coexistencia de más de treinta regímenes escalafonarios o sistemas de empleo diferentes para la Administración Central y más de sesenta en el ámbito de la Administración Pública Nacional. Esto incluye la existencia de cuerpos especiales estables. 
c) Criterios irracionales de selección de personal.

d) Asignación clientelar, corporativa o amiguista de los cargos de dirección en el aparato estatal y de los gastos reservados.

e) Nombramiento de no-idóneos en cargos directivos o de gran responsabilidad (Tesoro, 1999).

f) Concursos espurios. Para ilustrar esta cuestión, mi trabajo de investigación incluye el caso de la Dra. Susana Torrado, (único caso con nombre y apellido). En vez de entrevistarla, transcribí su denuncia pública de concurso espurio y el debate periodístico que entabló en septiembre de 1993, con quien era entonces Secretario de Programación Económica, el Lic. Juan Llach ${ }^{1}$.

g) Escandalosas desigualdades salariales aún para agentes que deben ser graduados universitarios y desarrollan funciones similares de organización, planeamiento y control, pero pertenecen a plantas permanentes y escalafones distintos. A este respecto pude publicar la escala salarial completa del escalafón SINAPA pero no fue tan sencillo transparentar los ingresos de otros escalafones permanentes como el de los Administradores Gubernamentales cuyos ingresos (información pública de circulación reservada) oscilan entre 4000 y 6000 pesos y triplican, injustificadamente, los ingresos de otros profesionales que cumplen similares funciones y deben reunir méritos académicos equivalentes. En el mismo sentido, Oszlak (2000) ha señalado que existen en el empleo público nacional y provincial, amplias variaciones en los valores pagados a funcionarios de nivel equivalente, según trabajen para el Ejecutivo, el Legislativo o el Judicial. Son incomprensibles los tratamientos diferenciales entre personal permanente y contratado y también otras graves distorsiones salariales resultantes del "nepotismo político".

h) Existencia de regímenes de privilegio y de onerosas consultorías financiadas por organismos internacionales, que contribuyen a aumentar la deuda externa.

i) Personal contratado como trabajadores autónomos, aún cuando la naturaleza de su prestación no condice con tal condición.

j) Sistema de capacitación obligatoria con "clientela cautiva" sólo para algunos escalafones. Los profesionales del escalafón SINAPA, estaban obligados a realizar cursos de capacitación por debajo de su nivel académico para ascender en la carrera administrativa (des-capacitación para ascender). A partir del Decreto 889/2001 el modelo de capacitación vigente en la Administración Pública Nacional ha sido reestructurado. El Instituto Nacional de la Administra-

1 Véase el concepto de "violencia transgresora" en Puget et al (1993). La definición conceptual y el análisis se realizó sobre la base de un caso cuyo protagonista ganó un concurso en la Administración Pública Argentina y no obtuvo el cargo legítimamente conquistado. 
ción Pública, INAP, pasa de cumplir su rol tradicional de proveedor de capacitación a ser el órgano rectorde un Sistema Nacional de Capacitación.

k) Desaliento a la excelencia profesional mediante criterios perversos de equivalencias para la capacitación externa al Instituto Nacional de la Administración Pública.

l) Evaluaciones de desempeño del personal con "cupos". Los "cupos" son las cantidades preestablecidas de calificaciones a distribuir entre su personal a cargo, que tiene el evaluador. Este procedimiento desvirtúa, de plano, el proceso de evaluación objetiva del desempeño del servidor público.

m) Becas de estudio - incluidas maestrías costosas - no difundidas y reservadas sólo para los amigos/as de los jefes de turno.

n) "Unidades retributivas" (UR) distribuidas a criterio del jefe político. Estas UR son sobresueldos o ingresos cuya denominación re-rotulada en términos de UR oculta el ingreso efectivamente percibido por el beneficiario, ya que cada unidad retributiva equivale a $\$ 2,80$ pesos argentinos.

o) Sumarios injustificados.

p) Desprecio y destrucción de la producción elaborada in situ por los agentes y carencia de Archivos y Catálogos de la Producción Documental generada por los servidores públicos.

q) Infrautilización de capacidades individuales.

r) Restricción a los agentes de la información más elemental para desarrollar su tarea. s) Hacinamiento laboral y Condiciones y Medio Ambiente de Trabajo degradantes. A título ilustrativo un juez, en "Carta Abierta" publicada en una revista de circulación semanal, declara que el juzgado donde desempeña sus tareas carece de instalaciones sanitarias (baño) y de agua corriente (Niño et al, 2001).

t) Inadecuación del mobiliario y falta de lugares destinados para el almuerzo de los trabajadores que comen sobre el escritorio, atendiendo, a veces, simultáneamente al público.

u) "Techo de cristal" para las mujeres. Límite invisible por encima del cual, las mujeres no pueden ascender en una pirámide jerárquica, sea académica, laboral, política o de otra índole (Burín, 1997 y Revista TEMAS, 1998).

v) Desprotección a la maternidad más allá de las declaraciones y aún de las "leyes". A título ilustrativo, un fallo de 1997 de la Corte Suprema de la Nación niega la licencia especial por parto a una empleada del Poder Judicial de la Nación que dio a luz en término a un hijo nacido muerto. Conforme con el fallo, la Justicia Nacional no contempla una solución explícita para los casos de alumbramiento sin vida (Mercado, 2000). También el mismo Estado propicia actitudes discriminatorias en la interpretación del Decreto 92/95 (régimen de contratos) de aplicación general. Recientemente, la Administración Pública colocó a seis embarazadas, contratadas bajo ese régimen, ante la férrea opción de abortar o perder el trabajo (Scialpi, 2001a). 
w) Acoso sexual como salvoconducto para conseguir o mantener puestos de trabajo.

x) Falta de respuesta (ausencia) del obligado pronunciamiento administrativo frente a los reclamos (administrativos) presentados por los agentes.

y) Recortes salariales practicados por decreto del Poder Ejecutivo Nacional. El primer recorte por Decreto $430 / 2000$ redujo el $12 \%$ en los salarios del Poder Ejecutivo Nacional (PEN) superiores a los $\$ 1000$. El segundo recorte de salarios de estatales por decreto 896/2001 incrementa el recorte y lo lleva al $13 \%$.

z) Amenazas de toda índole (incluida la de asesinar a la víctima) EI Síndico General de la Nación, fue amenazado de muerte. Dichas amenazas obedecen a los informes de la Sindicatura General de la Nación (SIGEN) sobre la malversación de fondos de la Secretaría de Inteligencia del Estado (SIDE) El operativo del asesinato fue informado a la víctima por fax (Gruss, 2001).

El procedimiento de la re-rotulación es tan habitual, que termina normalizado y naturalizado. Se re-significan las conductas y se hace de esta re-significación, algo normal y natural. La resignificación es un modo de justificar lo injustificable.

En este contexto, se llega a calificar de "persona equilibrada" o "persona que sabe adaptarse a las circunstancias " (entendido como elogio) a la persona que no cuestiona las abiertas violaciones a las normativas vigentes, ni mucho menos las arbitrariedades, algunas de ellas incluidas (avaladas y consolidadas) en las mis- mas normativas vigentes. Las arbitrariedades pueden ser ilegales o aún legales.

El aprendizaje social le impone al agente respetar a su superior, lo que conlleva la ideología (creencia) de que tiene la obligación de complacerlo, subordinándose a todo capricho o impulso del superior. Implícita e históricamente muchos agentes (¿la mayoría?) han incorporado este "deber de complacer". Esto significa no discutir en situaciones de trabajo y aceptar las re-rotulaciones (resignificaciones) de las conductas que realizan los jefes. El esquema de obediencia se construye diariamente en cada uno de nosotros, prestando "conformidad automática" según la denominación de Erich Fromm (1984).

La población que integra la Administración, desempeña sus tareas afectada de desamparo aprendido. Esta indefensión, impotencia o desamparo aprendido, es uno de los efectos más graves ocasionados a las víctimas de situaciones habituales de violencia (Scialpi, 1999 y 2000). Otros efectos no menos graves son el "Síndrome de Estocolmo" (Sluzky, 1994) y el "Trastorno de Mediocridad Inoperante Activa” -síndrome MIA- de acuerdo a la conceptualización de Gonzalez de Rivera y Revuelta (1997; 18 (6):229-231).

Bajo este tipo de circunstancias y de acuerdo a Pinkola Estes (1998), nuestro instinto de defensa es mutilado, perdemos la capacidad de huir y de defendernos e incumplimos nuestros compromisos de ayuda evitando los comportamientos valientes. De esta manera se encubre y se perpetúa la existencia y el funcionamiento de las relaciones violentas más frecuentes y cotidianas de nuestra sociedad, donde, según Izaguirre 
(1998) no se ve el ejercicio de la fuerza material y por eso ha sido naturalizada, normalizada. Cabe preguntarse, entonces, si estamos ante violencias menores, en comparación con otras violencias sociales, aunque se impone dudarlo frente a importantes "hallazgos anómalos" referidos a tasas de mortalidad y tasas de enfermedad específicas del sector (Evans y Marmor, 1996).

Esta violencia no-accidental requiere de un contexto en el cual algunos miembros del sistema tienen el poder de decidir - poner en acto - qué es lo que va a ser validado como "real" para todos los miembros del sistema administrativo público. Por lo mismo, la persona (se trate de profesionales, científicos, técnicos, empleados administrativos o personal de maestranza) es negada o invalidada en tanto "sujeto social" y es tratada como "objeto social". Cuando ocurre esto, cuando las instituciones que deben proteger, causan daño, lo hacen de una manera tal, que la violencia y el daño quedan mistificados y justificados, llegando incluso a desfigurar el Estado de Derecho.

Deseo reiterar aquí una observación realizada en otros espacios. A mi juicio, reservar el término violencia laboral para tipificar sólo al acoso sexual contra las mujeres, como lo hace el Primer Convenio Colectivo de Trabajo General para la Administración Pública Nacional Argentina empobrece demasiado el análisis y constituye una reducción inadmisible en la práctica laboral. En tal sentido, a juzgar por lo que sostiene el artículo 137 del Convenio - (Decreto 66/99 Boletín Oficial $26 / 2 / 99$ ) - con vigencia a partir del 1 ro. de enero de 1999, sólo el acoso sexual con- tra las mujeres es violencia laboral. Cabe preguntar a los redactores y firmantes del Convenio, bajo qué otra denominación agruparían las situaciones que he identificado y enumerado y que padecen tanto mujeres como varones de la Administración Pública Argentina.

Por último, realizaré dos consideraciones adicionales. La primera: Para Hirigoyen (1999) el mayor obstáculo que enfrenta el fenómeno del acoso moral es la dificultad que existe para hallar y organizar "pruebas flagrantes". Por el contrario, para el fenómeno que visibilicé - la violencia político-burocrática - a mi juicio, resulta relativamente fácil encontrar pruebas contundentes. Hay evidencias escritas - informaciones oficiales, normativas vigentes, expedientes, documentos, acuerdos de la Corte Suprema de Justicia y hasta páginas oficiales de información en Internet que ofrecen testimonios elocuentes de lo que ocurre. Un testimonio elocuente de esto, es el listado de beneficiarios de "jubilaciones de privilegio" (para las cuales los beneficiarios no necesitan reunir ni la edad ni el tiempo requerido para jubilarse normalmente y por añadidura la reciben en simultáneo con otros jubilados reglamentarios) que aparece en la página web de la Procuración del Tesoro de la Nación (www.ptn.gov.ar, 12/09/01). Esto sucede en momentos en que el gobierno aplica el "recorte" a los ingresos de jubilados a partir de los $\$ 500$. La violencia (padecida y sentida) que emerge de tal iniquidad es de interpretación subjetiva y la iniquidad es absolutamente legal. La segunda consideración: He señalado que no hay mayor dificultad para hallar pruebas flagrantes de violencia político-burocrática. Pero corres- 
ponde distinguir entre violencias ilegales (que violan la normativa) y violencias legales (prebendas, privilegios e irracionalidades contenidas en la normativa vigente). En consecuencia y por último, ambos fenómenos - acoso moral y violencia político-burocrática - (institucional) son complementarios. En la mayoría de los casos que investigué, las situaciones de violencia político-burocrática de verificación probada, según el trabajo de campo (aunque no suficientemente visibilizadas y menos aún denunciadas) fueron padecidas conjuntamente con prácticas de acoso moral, mobbing u hostigamientos de carácter psicológico mucho más difíciles de probar.

\section{Conclusiones}

Más allá de la gravedad que pueda traducir esta enunciación del fenómeno de la violencia político-burocrática, los aspectos señalados han sido escasos para describir una temática vasta que requiere rigurosa investigación, denuncia sistemática, prevención y atención pública. Quedan pendientes de tratamiento más amplio, la funcionalidad del fenómeno para el desarrollo de la corrupción (Scialpi, 2001a) y los impactos sobre la salud de los servidores públicos. Sobre éstos, un antecedente invalorable es el trabajo de Carlos Aníbal Rodríguez (1990) que analizó la situación de los trabajadores en la Argentina en quince sectores de actividad. El trabajo no incluyó al sector público.

Confío en que lo apuntado bastará para llamar la atención sobre un fenómeno invisibilizado, silenciado y naturalizado hasta el extremo. A fuerza de tanta ceguera, de tanta "ilegalidad masiva" y "anomia boba" (Nino, 1992:43) la Argenti- na enfrenta hoy una crisis institucional terminal. Por cierto, las víctimas de violencia laboral en la función pública, no encuentran un cauce apropiado para presentar reclamos, ni menos aún legislación (sí proyectos de ley) que proteja a los denunciantes y sancione a los victimarios (Berhongaray, 1998 y Carca, 2001).

Confío en que este relevamiento mínimo de problemas -considerados urgentes para los servidores públicos- resulte útil a la hora de formular prioridades de investigación por parte de quienes estamos involucrados con el tema de la violencia institucional. Desde luego aspiro haber propiciado en los lectores la interpretación subjetiva de que la situación es realmente grave e indeseable. Más aún, espero haberlos motivado a la consideración de que algo podemos y debemos hacer para prevenirla, corregirla y atenderla. Algunas iniciativas ya están en marcha (Scialpi, 2001 b).

Están en juego nuestra salud pública, nuestros derechos humanos, nuestro desarrollo humano y nuestro Estado de Derecho. A todos -individuos, gobiernos, organizaciones no gubernamentales, empresas, dirigentes políticos, organizaciones multilaterales- corresponde un papel de transformación "que fomente auténticamente las libertades fundamentales en todas partes, en lugar de rendirles homenaje de los dientes para afuera" (PNUD, 2000:1) Concluyo con el mismo sentimiento de la juglaresa Blázquez (1995) "porque me duele que sea cierto, con dolor del más profundo" y creo necesario alegar que no es tiempo de permanecer quietos mirando pasar las cosas que nos ocurren. Deseo contribuir activamente a cambiar este estado de situación. 


\section{Bibliografía Citada}

Almada López, Carlos (2001), “Gobernabilidad e Ingobernabilidad conceptos básicos". Documento disponible en el Sistema Integrado y Analítico de Información sobre Reforma del Estado, Gestión y Políticas Públicas (SIARE) del Centro Latinoamericano de Administración para el Desarrollo. http:// www.clad.org.ve

Araujo, Kathia (2000), "El surgimiento de la violencia doméstica como problema público y objeto de políticas". Revista de la CEPAL, No 70. Santiago de Chile. Naciones Unidas.

Blázquez, Eladia (1995), “Argentina Primer Mundo" (canción) en "Con las alas del alma." - CD 51339.SADAIC. Argentina.

Boletín Oficial de las Cortes Generales (2001), Serie B - Número 175-1 y 176-1. España.

Burin, Mabel (1997), "Género y Psicoanálisis: Subjetividades Femeninas Vulnerables", en Foro de Discusión sobre Psicoanálisis, Estudios Feministas y Género. www.psiconet.com./foros/genero.

Carca, Elisa (2001), Proyecto de Violencia Laboral. Provincia de Buenos Aires. Honorable Cámara de Senadores de la Provincia de Buenos Aires.

Coria, Clara (1994), Las negociaciones nuestras de cada día. Buenos Aires, Editorial Piados.

Corsi, Jorge (compilador) (1994), Violencia Familiar. Una mirada interdisciplinaria sobre un grave problema social. Buenos Aires, Editorial Piados.

Chappell y Di Martino (1998), Violence at work, Ginebra, Organización Internacional del Trabajo, OIT.

Evans, Barer y Marmor (1996), ¿Por qué alguna gente está sana y otra no? Ma- drid, España, Editorial Díaz de Santos.

Filho, Pellegrini Alberto (1999), "La violencia y la salud pública”, en Revista Panamericana de Salud Pública. Número especial sobre la Violencia. Vol. 5 Nos. 4/5, Washington DC, U.S.A, Organización Panamericana de Salud.

Fournier, M., De los Ríos, R., Orpinas, P. y Piquet-Carneiro, L. (1999), "Estudio Multicéntrico sobre Actitudes y Normas Culturales frente a la Violencia (proyecto ACTIVA) Metodología", en Revista Panamericana de Salud Pública. Vol. 5. Nos. 4/5, Volumen especial sobre la violencia. Washington DC, U.S.A.

Fromm, Erich (1984), EI Miedo a la Libertad. México, Editorial Paidós.

Godelier, Maurice (1984), L' idéel et le matériel. París. Fayard.

González de Rivera y Revuelta, J. L. (1997), "El Trastorno por Mediocridad Inoperante Activa (síndrome MIA)". Psiquis,(6): 229.231. España.

Gore, Al (1995), Un gobierno más efectivo y menos costoso. Edamex, Méjico.

Gruss, Luis (2001), “¿Por qué quieren matar a este hombre?" en Revista 3 Puntos. Número 203. (Mayo 17) Buenos Aires.

Hirigoyen, Marie France (1999), El acoso Moral: el maltrato psicológico en la vida cotidiana, Barcelona, Editorial Paidós.

Hirigoyen, Marie France (2001), El acoso moral en el Trabajo. Buenos Aires. Editorial Paidós.

Horton, R.L. (1987), Problemas Sociales. Buenos Aires. Editorial El Ateneo.

Izaguirre, Inés (1998), "El Poder en proceso: La violencia que no se ve", en Emir Sader Democracia sin exclusiones ni excluidos. Venezuela, Editorial Nueva Sociedad. 
Mercado, Matilde (2000), "La división sexual del trabajo: permanencias y cambios" en Birgin, Haydée. Ley, Mercado y Discriminación. El Género del Trabajo. Buenos Aires, Editorial Biblos.

Nigro, Lloyd and Waugh, William (1996), "Violence in the American workplace: Challenges to the Public Employer." Public Administration Review, Volume 56, Numer 4. Estados Unidos.

Nino, Carlos (1992), Un país al margen de la Ley. Buenos Aires. Editorial Emecé.

Niño Carlos (2001), "Carta Abierta”, en Revista Tres Puntos, abril 27. Buenos Aires. Argentina.

O'Donnell, Guillermo (1997), Entrevista de J. Halperín. Diario Clarín. Argentina.

O'Donnell, Guillermo (2000), "Vale la pena aprender a oir a la ciudadanía". Entrevista de Astrid Pikielny.Diario La Nación. Argentina.

O'Donnell, Horacio (1999), La Violencia en el Sistema Educativo. Buenos Aires, Editorial Temas.

Oszlak, Oscar (1994), "Los Administradores Gubernamentales. La creación de un Cuerpo Gerencial de Elite en el Sector Público Argentino" Informe a la Conferencia del Banco Mundial sobre Reforma del Servicio Civil en América Latina y el Caribe, Washington, D.C. Mayo 1993. En: Revista APORTES de la A.A.G. Año 1, número 1, Buenos Aires.

Oszlak, Oscar (2000), "El mito del Estado Mínimo: Una década de Reforma Estatal en la Argentina", trabajo presentado al $V$ Congreso Internacional del CLAD sobre Reforma del Estado y de la Administración Pública, Versión Preliminar (inédita).

Pinkola Estes, Clarissa (1998), Mujeres que corren con los lobos. Ediciones B, Barcelona.
Primer Convenio Colectivo de Trabajo General para la Administración Pública Nacional Argentina. Decreto 66/99 Boletín Oficial 26/2/99.

Procuración del Tesoro de la Nación Argentina. Página Web. http://www.ptn.gov.ar 12/09/01.

Programa de las Naciones Unidas para el Desarrollo (PNUD) (2000), Informe Sobre Desarrollo Humano. Madrid, Ediciones Mundi-Prensa.

Puget, Janine; Bianchedi, E.T., Bianchedi, M ; Braun, J.y Pelento M.L. (1993), "Violencia Social Transgresora", en Gaceta Psicológica. Número 94, Marzo/Abril, Argentina. Asociación de Psicólogos de Buenos Aires.

Revista Derecho del Trabajo (2000), Año LXnúmero 10. Argentina.

Revista TEMAS, Estado, Administración y Políticas Públicas (1995), Año 1. No.3 No figura el autor. "Informe sobre Empleo Público". Instituto Nacional de la Administración Pública, INAP, Argentina.

Revista TEMAS, Estado, Administración y Políticas Públicas (1998), Año 4. No. 14. No figura el autor. "El techo de cristal en el empleo público femenino: algunas características observadas en el SINAPA" Argentina. Instituto Nacional de la Administración Pública, INAP.

Rodríguez, Carlos A. (1990), Salud y Trabajo. La Situación de los Trabajadores en la Argentina. Latina. Buenos Aires. Centro Editor de América.

Sánchez Carazo, Carmen (2001), "Llorar de impotencia”, Diario El País, España, 11/9/2001 http://www.elpais.es/articulo.html

Scialpi, Diana (1999), Violencias en la Administración Pública: Casos y Miradas para pensar la Administración Pública Nacional como ámbito laboral. Buenos Aires, Editorial Catálogos. 
Scialpi, Diana (2000), "Habituados a soportar la violencia"; "Sometidos al acoso moral" y "Planes contra la Violencia en el Trabajo". Columna Miradas, Suplemento Empleos (dominical) del Diario La Nación.

Scialpi, Diana (2001a), "La violencia en la gestión de personal de la función pública, como condición necesaria para el desarrollo de la corrupción". Revista Probidad No.14. dedicada a la corrupción en la Argentina. http://www.probidad.org.sv/revista.

Scialpi, Diana (2001b), 'La violencia laboral en la Administración Pública Central Argentina. Impactos y Funcionalidad." Trabajo presentado al VI Congreso Internacional del CLAD sobre Reforma del Estado y la Administración Pública, Buenos Aires. Versión en CD. (ISBN: 980-6125-38-X) y disponible en

http:// www.clad.org.ve/docume.html
SIEMPRO-INDEC (2000), Encuesta de Desarrollo Social y Condiciones de Vida. Datos de 1997. Resultados definitivos del nivel nacional. Sistema de Información y Evaluación y Monitoreo Programas Sociales (SIEMPRO) e Instituto Nacional de Estadística y Censos, (INDEC) Argentina.

Sluzky, Carlos (1994), "Violencia Familiar y Violencia Política: Implicancias terapéuticas de un modelo general", en: Nuevos Paradigmas y Subjetividades, Editorial Paidós. Buenos Aires.

Tesoro, J.L. (1999), "Reflexiones acerca de la Profesionalización del Servicio Civil". Revista TEMAS, Estado, Administración y Políticas Públicas. Año 5. No. 17. p 15-30. Argentina. Instituto Nacional de la Administración Pública, INAP.

Berhongaray, Antonio T. (1998) "Proyecto de Ley", Honorable Cámara de Senadores, Argentina, sin sanción. Expediente $1852 / 98$. 